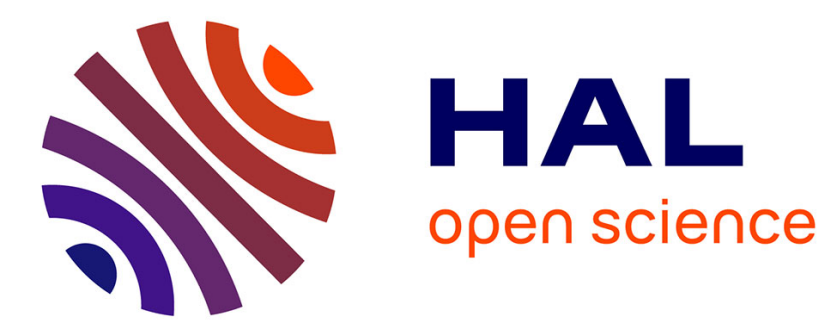

\title{
CP decomposition of semi-nonnegative semisymmetric tensors based on QR matrix factorization
}

Lu Wang, Laurent Albera, Amar Kachenoura, Huazhong Shu, Lotfi Senhadji

\section{To cite this version:}

Lu Wang, Laurent Albera, Amar Kachenoura, Huazhong Shu, Lotfi Senhadji. CP decomposition of semi-nonnegative semisymmetric tensors based on QR matrix factorization. The eighth IEEE Sensor Array and Multi-Channel Signal Processing Workshop, Jun 2014, A Coruna, Spain. 4 p. hal-01012125

\section{HAL Id: hal-01012125 \\ https://hal.science/hal-01012125}

Submitted on 25 Jun 2014

HAL is a multi-disciplinary open access archive for the deposit and dissemination of scientific research documents, whether they are published or not. The documents may come from teaching and research institutions in France or abroad, or from public or private research centers.
L'archive ouverte pluridisciplinaire HAL, est destinée au dépôt et à la diffusion de documents scientifiques de niveau recherche, publiés ou non, émanant des établissements d'enseignement et de recherche français ou étrangers, des laboratoires publics ou privés. 


\title{
CP decomposition of semi-nonnegative semi- symmetric tensors based on QR matrix factorization
}

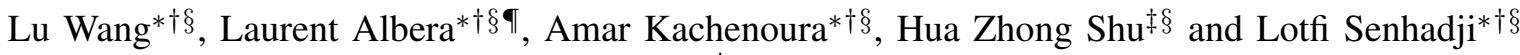 \\ *INSERM, UMR 1099, Rennes, F-35000, France †Université de Rennes 1, LTSI, Rennes, F-35000, France \\ ${ }^{\S}$ Centre de Recherche en Information Biomédicale sino-français (CRIBs), Rennes, France \\ $\ddagger$ LIST, Southeast University, 2 Sipailou, 210096, Nanjing, China \\ IINRIA, Centre Inria Rennes - Bretagne Atlantique, 35042 Rennes, France. \\ Email: wangLyu1986@hotmail.com
}

\begin{abstract}
The problem of Canonical Polyadic (CP) decomposition of semi-nonnegative semi-symmetric three-way arrays is often encountered in Independent Component Analysis (ICA), where the cumulant of a nonnegative mixing process is frequently involved, such as the Magnetic Resonance Spectroscopy (MRS). We propose a new method, called $\mathrm{JD}_{\mathrm{QR}}^{+}$, to solve such a problem. The nonnegativity constraint is imposed by means of a square change of variable. Then the high-dimensional optimization problem is decomposed into several sequential rational subproblems using QR matrix factorization. A numerical experiment on simulated arrays emphasizes its good performance. A BSS application on MRS data confirms the validity and improvement of the proposed method.
\end{abstract}

\section{INTRODUCTION AND PROBLEM FORMULATION}

Canonical Polyadic (CP) decomposition of a multi-way array [1]-[3] plays an important role in Blind Source Separation (BSS), particularly in Independent Component Analysis (ICA) [4]. In this paper, we consider the following semi-nonnegative semi-symmetric CP decomposition problem:

Problem 1. The semi-nonnegative semi-symmetric CP decomposition of a 3-way array $\mathcal{C} \in \mathbb{R}^{N \times N \times K}$, is the minimal linear combination of rank-1 3-way arrays that yields $\mathcal{C}$ exactly:

$$
\mathcal{C}=\sum_{p=1}^{P} \boldsymbol{a}_{p} \circ \boldsymbol{a}_{p} \circ \boldsymbol{d}_{p}
$$

subject to $\boldsymbol{A}=\left[\boldsymbol{a}_{1}, \cdots, \boldsymbol{a}_{P}\right] \in \mathbb{R}^{N \times P}$ having nonnegative components, where o denotes the outer product. $\boldsymbol{A}$ and $\boldsymbol{D}=$ $\left[\boldsymbol{d}_{1}, \cdots, \boldsymbol{d}_{P}\right] \in \mathbb{R}^{K \times P}$ are called the loading matrices of $\mathcal{C}$. $P$ is then the rank of $\mathcal{C}$.

The decomposition is considered to be essentially unique when the uniqueness is guaranteed up to scaling and permutation indeterminacies. This problem is often encountered in ICA when a nonnegative mixing matrix is considered. For example, in Magnetic Resonance Spectroscopy (MRS), the mixing matrix contains the positive concentrations of the source metabolites. Then the 3-way array built by stacking the matrix slices of a cumulant is both nonnegative and symmetric in two modes. Equation (1) can also be described by using the frontal slices of $\mathcal{C}: \boldsymbol{C}^{(k)}=\mathcal{C}_{:,:, k}=\boldsymbol{A} \boldsymbol{D}^{(k)} \boldsymbol{A}^{\top}, \forall k \in\{1,2, \cdots, K\}$, where $\boldsymbol{D}^{(k)} \in \mathbb{R}^{P \times P}$ is a diagonal matrix whose diagonal contains the elements of the $k$-th row of $\boldsymbol{D}$, and $\boldsymbol{C}^{(k)} \in \mathbb{R}^{N \times N}$ is the $k$-th frontal slice $\mathcal{C}$. In this paper, we focus on computing the square matrix $\boldsymbol{A}$, where $N=P$. In order to compute
$\boldsymbol{A}$, we can resort to solve the following nonnegative Joint Diagonalization by Congruence (JDC) problem:

Problem 2. Given a 3-way array $\mathcal{C} \in \mathbb{R}^{N \times N \times K}$ with $K$ symmetric frontal slices $\boldsymbol{C}^{(k)} \in \mathbb{R}^{N \times N}$, find a matrix $\boldsymbol{A} \in$ $\mathbb{R}^{N \times N}$ and $K$ diagonal matrices $D^{(k)} \in \mathbb{R}^{N \times N}$ such that:

$$
\forall k \in\{1,2, \cdots, K\}, \boldsymbol{C}^{(k)}=\boldsymbol{A} \boldsymbol{D}^{(k)} \boldsymbol{A}^{\top}
$$

subject to $\boldsymbol{A}$ having nonnegative components.

Many existing CP algorithms handle the symmetry and the nonnegativity separately, such as in [5]-[7]. Several methods consider the combination of both constrains [8], [9], but they aim at solving different problems rather than problem 1. Only a few methods were proposed to solve the nonnegative JDC problem [10], [11]. In this paper, we propose a new algorithm, called $\mathrm{JD}_{\mathrm{QR}}^{+}$, based on minimizing the following indirect least square criterion [6], [12]:

$$
J_{1}(\boldsymbol{A})=\sum_{k=1}^{K} \| \text { off }\left(\boldsymbol{A}^{-1} \boldsymbol{C}^{(k)} \boldsymbol{A}^{-\mathrm{T}}\right) \|_{F}^{2}
$$

where off(.) vanishes the diagonal components of the input matrix, the superscript ${ }^{-T}$ denotes the inverse of the transposed matrix, and $\|.\|_{F}$ computes the Frobenius norm. The nonnegativity constraint is imposed by means of a square change of variable. The $\mathrm{QR}$ matrix factorization of the Hadamard square root of $\boldsymbol{A}$ decomposes the high-dimensional optimization problem into a sequential rational subproblems. In addition, the rotation matrix and the unit triangular matrix of the QR factorization have unit determinants, therefore the resulting matrix $\boldsymbol{A}$ is nonsingular. A numerical experiment on simulated arrays emphasizes its good performance. A BSS application on MRS data confirms the validity and improvement of the proposed method.

\section{THE JD $\mathrm{QR}_{\mathrm{QR}}^{+}$METHOD}

In order to avoid the inverse of $\boldsymbol{A}$ in cost function (3), let us consider the following assumptions: $\boldsymbol{i}) \boldsymbol{A} \in \mathbb{R}_{+}^{N \times N}$ is nonsingular; ii) $\boldsymbol{D} \in \mathbb{R}^{K \times N}$ is nonsingular and does not contain zero entries. Then each frontal slice of $\mathcal{C}$ is nonsingular and its inverse can be expressed as follows:

$$
\left(\boldsymbol{C}^{(k)}\right)^{-1}=\boldsymbol{A}^{-\top}\left(\boldsymbol{D}^{(k)}\right)^{-1} \boldsymbol{A}^{-1}
$$

In practice, only the sufficiently well-conditioned matrix $\boldsymbol{C}^{(k)}$ is chosen when its condition number is below a predefined 
threshold. We use $\boldsymbol{C}^{(k,-1)}$ to denote $\left(\boldsymbol{C}^{(k)}\right)^{-1}$ for simplicity. Equation (4) shows that $\boldsymbol{C}^{(k,-1)}$ is jointly diagonalizable by $\boldsymbol{A}$. Then $\boldsymbol{A}$ can be estimated by minimizing the following modified criterion of (3) directly:

$$
J_{2}(\boldsymbol{A})=\sum_{k=1}^{K} \| \text { off }\left(\boldsymbol{A}^{\top} \boldsymbol{C}^{(k,-1)} \boldsymbol{A}\right) \|_{F}^{2}
$$

The nonnegativity constraint on $\boldsymbol{A}$ can be imposed by a square change of variable: $\boldsymbol{A}=\boldsymbol{B} \odot \boldsymbol{B}=\boldsymbol{B}^{\square 2}$, where $\boldsymbol{B} \in \mathbb{R}^{N \times N}$ and where $\square$ denotes Hadamard product [13], [14]. Then we can find $\boldsymbol{A} \in \mathbb{R}_{+}^{N \times N}$ by estimating $\boldsymbol{B} \in \mathbb{R}^{N \times N}$, such that $\boldsymbol{A}=\boldsymbol{B}^{\square 2}$, and $\boldsymbol{B}$ is the global minimum of the following cost function:

$$
J_{2}(\boldsymbol{B})=\sum_{k=1}^{K} \| \text { off }\left(\left(\boldsymbol{B}^{\square 2}\right)^{\top} \boldsymbol{C}^{(k,-1)} \boldsymbol{B}^{\square 2}\right) \|_{F}^{2}
$$

Now let us recall the following definitions and lemmas:

Definition 1. A unit upper triangular matrix is an upper triangular matrix whose main diagonal entries are 1 .

Definition 2. An elementary upper triangular matrix $\boldsymbol{R}^{(i, j)}\left(r_{i, j}\right)$ is equal to an identity matrix except the $(i, j)$-th entry, which is equal to $r_{i, j}$.

Definition 3. A Givens rotation matrix $\boldsymbol{Q}^{(i, j)}\left(\theta_{i, j}\right)$ is equal to an identity matrix except the $(i, i)$-th, $(j, j)$-th, $(i, j)$-th and $(j, i)$-th entries, which are equal to $\cos \left(\theta_{i, j}\right), \cos \left(\theta_{i, j}\right)$, $-\sin \left(\theta_{i, j}\right)$ and $\sin \left(\theta_{i, j}\right)$, respectively.

Lemma 1. Any $(N \times N)$ unit upper triangular matrix can be factorized as a product of $N(N-1) / 2$ elementary upper triangular matrices.

Lemma 2. Any $(N \times N)$ orthonormal matrix can be factorized as a product of, at most, $N(N-1) / 2$ Givens rotation matrices.

For any nonsingular matrix $\boldsymbol{B} \in \mathbb{R}^{N \times N}$, the $\mathrm{QR}$ matrix factorization decomposes it as $\boldsymbol{B}=\boldsymbol{Q} \boldsymbol{R} \boldsymbol{\Lambda}$, where $\boldsymbol{Q} \in \mathbb{R}^{N \times N}$ is a orthonormal matrix, $\boldsymbol{R} \in \mathbb{R}^{N \times N}$ is a unit upper triangular matrix, and $\Lambda \in \mathbb{R}^{N \times N}$ is a diagonal matrix. Due to the indeterminacies of the $\mathrm{CP}$ decomposition, the matrix $\boldsymbol{B}$ solving (6) can be chosen as $\boldsymbol{B}=\boldsymbol{Q R}$ without loss of generality. Moreover, lemma 1 and lemma 2 yield that $\boldsymbol{B}$ can be written as a product of the following matrices:

$$
\boldsymbol{B}=\prod_{i=1}^{N} \prod_{j=i+1}^{N} \boldsymbol{Q}^{(i, j)}\left(\theta_{i, j}\right) \prod_{i=1}^{N} \prod_{j=i+1}^{N} \boldsymbol{R}^{(i, j)}\left(r_{i, j}\right)
$$

As a consequence, the minimization of (6) with respect to $B$ is converted to the estimation of $N(N-1)$ parameters: $\theta_{i, j}$ and $r_{i, j}$. We propose a Jacobi-like procedure, called $\mathrm{JD}_{\mathrm{QR}}^{+}$, in order to compute these parameters sequentially.

\section{A. Minimization with respect to $\boldsymbol{Q}^{(i, j)}\left(\theta_{i, j}\right)$}

Let $\tilde{\boldsymbol{A}}$ and $\tilde{\boldsymbol{B}}$ denote the current estimate of $\boldsymbol{A}$ and $\boldsymbol{B}$ before estimating $\boldsymbol{Q}^{(i, j)}\left(\theta_{i, j}\right)$, respectively. Let $\tilde{\boldsymbol{A}}^{(\text {new })}$ and $\tilde{\boldsymbol{B}}^{\text {(new) }}$ stand for $\tilde{\boldsymbol{A}}$ and $\tilde{\boldsymbol{B}}$ updated by $\boldsymbol{Q}^{(i, j)}\left(\theta_{i, j}\right)$, respectively. Furthermore, the update of $\tilde{\boldsymbol{B}}$ is defined as follows:

$$
\tilde{\boldsymbol{B}}^{\text {(new) }}=\tilde{\boldsymbol{B}} \boldsymbol{Q}^{(i, j)}\left(\theta_{i, j}\right)
$$

In order to compute $\theta_{i, j}$, the natural way is to minimize criterion (6) with respect to $\theta_{i, j}$ by replacing matrix $\tilde{\boldsymbol{B}}$ by $\tilde{\boldsymbol{B}}^{\text {(new) }}$. For the sake of convenience, we denote $J_{2}\left(\theta_{i, j}\right)$ instead of $J_{2}\left(\tilde{\boldsymbol{B}}^{\text {(new) }}\right) . J_{2}\left(\theta_{i, j}\right)$ can be expressed as follows:

$$
J_{2}\left(\theta_{i, j}\right)=\sum_{k=1}^{K}\left\|\operatorname{off}\left\{\left[\left(\tilde{\boldsymbol{B}}^{(\text {new })}\right)^{\square 2}\right]^{\top} \boldsymbol{C}^{(k,-1)}\left[\left(\tilde{\boldsymbol{B}}^{\text {(new) }}\right)^{\square 2}\right]\right\}\right\|_{F}^{2}
$$

The Hadamard square of $\tilde{\boldsymbol{B}}^{\text {(new) }}$ in (9) can be written as a function of $\theta_{i, j}$ as follows:

$\left(\tilde{\boldsymbol{B}}^{(\text {new })}\right)^{\square 2}=\tilde{\boldsymbol{B}}^{\square 2}\left(\boldsymbol{Q}^{(i, j)}\left(\theta_{i, j}\right)\right)^{\square 2}+\sin (2 \theta)\left(\tilde{\boldsymbol{b}}_{i} \square \tilde{\boldsymbol{b}}_{j}\right)\left(\boldsymbol{e}_{i}^{\boldsymbol{\top}}-\boldsymbol{e}_{j}^{\boldsymbol{\top}}\right)$

where $\tilde{\boldsymbol{b}}_{i}$ denotes the $i$-th column of $\tilde{\boldsymbol{B}}$, and $\boldsymbol{e}_{i}$ is the $i$-th column of the identity matrix $\boldsymbol{I} \in \mathbb{R}^{N \times N}$. Inserting (10) into the cost function (9), we obtain:

$$
\begin{aligned}
& J_{2}\left(\theta_{i, j}\right)=\sum_{k=1}^{K} \| \text { off }\left(\tilde{\boldsymbol{C}}^{(k, \text { new })}\right) \|_{F}^{2} \\
= & \sum_{k=1}^{K} \| \text { off }\left(\left[\left(\boldsymbol{Q}^{(i, j)}\left(\theta_{i, j}\right)\right)^{\square 2}\right]^{\top} \tilde{\boldsymbol{C}}^{(k)}\left(\boldsymbol{Q}^{(i, j)}\left(\theta_{i, j}\right)\right)^{\square 2}\right. \\
& +\sin (2 \theta)\left[\left(\boldsymbol{Q}^{(i, j)}\left(\theta_{i, j}\right)\right)^{\square 2}\right]^{\top} \tilde{\boldsymbol{c}}^{(k, 1)}\left(\boldsymbol{e}_{i}^{\top}-\boldsymbol{e}_{j}^{\top}\right) \\
& +\sin (2 \theta)\left(\boldsymbol{e}_{i}-\boldsymbol{e}_{j}\right) \tilde{\boldsymbol{c}}^{(k, 2)}\left(\boldsymbol{Q}^{(i, j)}\left(\theta_{i, j}\right)\right)^{\square 2} \\
& \left.+\sin ^{2}(2 \theta) \tilde{c}^{(k, 3)}\left(\boldsymbol{e}_{i}-\boldsymbol{e}_{j}\right)\left(\boldsymbol{e}_{i}^{\top}-\boldsymbol{e}_{j}^{\top}\right)\right) \|_{F}^{2}
\end{aligned}
$$

where $\tilde{\boldsymbol{C}}^{(k)}=\tilde{\boldsymbol{A}}^{\top} \boldsymbol{C}^{(k,-1)} \tilde{\boldsymbol{A}}, \tilde{\boldsymbol{c}}^{(k, 1)}=\tilde{\boldsymbol{A}}^{\top} \boldsymbol{C}^{(k,-1)}\left(\tilde{\boldsymbol{b}}_{i} \boxminus \tilde{\boldsymbol{b}}_{j}\right)$, $\tilde{\boldsymbol{c}}^{(k, 2)}=\left(\tilde{\boldsymbol{c}}^{(k, 1)}\right)^{\top}$ and $\tilde{c}^{(k, 3)}=\left(\tilde{\boldsymbol{b}}_{i} \boxminus \tilde{\boldsymbol{b}}_{j}\right)^{\top} \boldsymbol{C}^{(k,-1)}\left(\tilde{\boldsymbol{b}}_{i} \sqcup \tilde{\boldsymbol{b}}_{j}\right)$ are a matrix, a column vector, a row vector and a scalar of constant values, respectively. (11) shows that just the $i$-th and $j$-th columns and rows of $\tilde{\boldsymbol{C}}^{(k, \text { new })}$ involve the parameter $\theta_{i, j}$. It is noteworthy that the $(i, j)$-th and $(j, i)$-th elements are twice affected by the transformation. Inspired by [12], we propose to minimize the sum of the squares of the $(i, j)$-th entries of the $K$ symmetric matrices $\tilde{C}^{(k \text {,new })}$, instead of minimizing all the off-diagonal entries. This simplified minimization criterion is denoted by $\tilde{J}_{2}\left(\theta_{i, j}\right)$. The $(i, j)$-th element of $\tilde{\boldsymbol{C}}^{(k, \text { new })}$ can be expressed as a function of $\theta_{i, j}$ as follows:

$$
\begin{aligned}
& \tilde{C}_{i, j}^{(k, \text { new })}=-\sin ^{2}\left(2 \theta_{i, j}\right) \tilde{c}^{(k, 3)} \\
& +\sin ^{2}\left(\theta_{i, j}\right)\left(\tilde{C}_{i, i}^{(k)} \cos ^{2}\left(\theta_{i, j}\right)+\tilde{C}_{j, i}^{(k)} \sin ^{2}\left(\theta_{i, j}\right)\right) \\
& +\cos ^{2}\left(\theta_{i, j}\right)\left(\tilde{C}_{i, j}^{(k)} \cos ^{2}\left(\theta_{i, j}\right)+\tilde{C}_{j, j}^{(k)} \sin ^{2}\left(\theta_{i, j}\right)\right) \\
& +\sin \left(2 \theta_{i, j}\right)\left(\tilde{c}_{i}^{(k, 1)} \cos ^{2}\left(\theta_{i, j}\right)+\tilde{c}_{j}^{(k, 1)} \sin ^{2}\left(\theta_{i, j}\right)\right) \\
& -\sin \left(2 \theta_{i, j}\right)\left(\tilde{c}_{j}^{(k, 2)} \cos ^{2}\left(\theta_{i, j}\right)+\tilde{c}_{i}^{(k, 2)} \sin ^{2}\left(\theta_{i, j}\right)\right)
\end{aligned}
$$

where $\tilde{C}_{i, j}^{(k)}$ is the $(i, j)$-th element of $\tilde{\boldsymbol{C}}^{(k)}$ and $\tilde{c}_{i}^{(k, q)}$ is the $i$-th element of vector $\tilde{\boldsymbol{c}}^{(k, q)}$ with $q \in\{1,2\}$. By using the Weierstrass change of variable: $t_{i, j}=\tan \left(\theta_{i, j}\right)$, the expression of (12) can be rewritten as follows:

$$
\tilde{C}_{i, j}^{(k, \text { new })}=\frac{f_{4}^{(k)} t_{i, j}^{4}+f_{3}^{(k)} t_{i, j}^{3}+f_{2}^{(k)} t_{i, j}^{2}+f_{1}^{(k)} t_{i, j}+f_{0}^{(k)}}{\left(1+t_{i, j}^{2}\right)^{2}}
$$

where $f_{4}^{(k)}=\tilde{C}_{j, i}^{(k)}, f_{3}^{(k)}=-2 \tilde{c}_{i}^{(k, 1)}, f_{2}^{(k)}=\tilde{C}_{i, i}^{(k)}+\tilde{C}_{j, j}^{(k)}+$ $2 \tilde{c}_{j}^{(k, 2)}-4 \tilde{c}^{(k, 3)}, f_{1}^{(k)}=2 \tilde{c}_{i}^{(k, 2)}-\tilde{c}_{j}^{(k, 1)}$ and $f_{0}^{(k)}=\tilde{C}_{j, j}^{(k)}$. Equation (13) shows that the sum of the squares of $\tilde{C}_{i, j}^{(k, \text { new })}$, is a rational function in $t_{i, j}$, namely $\tilde{J}_{2}\left(t_{i, j}\right)$, where the degrees of the numerator and the denominator are 8 and 8 , respectively. The global minimum $t_{i, j}$ can be obtained by computing the roots of its derivative and selecting the one yielding the smallest value of $\tilde{J}_{2}\left(t_{i, j}\right)$. Once $t_{i, j}$ is obtained, $\theta_{i, j}$ can be computed by $\theta_{i, j}=\arctan \left(t_{i, j}\right)$. Then $\tilde{\boldsymbol{B}}$ is updated by (8) and $\tilde{\boldsymbol{A}}$ is updated by computing $\left(\tilde{\boldsymbol{B}}^{(\text {new })}\right)^{\square 2}$. 


\section{B. Minimization with respect to $\boldsymbol{R}^{(i, j)}\left(r_{i, j}\right)$}

Let $\tilde{\boldsymbol{A}}$ and $\tilde{\boldsymbol{B}}$ continue to denote the current estimate of $\boldsymbol{A}$ and $\boldsymbol{B}$ before estimating $\boldsymbol{R}^{(i, j)}\left(r_{i, j}\right)$, respectively. The update of $\tilde{\boldsymbol{B}}$, denoted by $\tilde{\boldsymbol{B}}^{\text {(new) }}$, is defined as follows:

$$
\tilde{\boldsymbol{B}}^{\text {(new) }}=\tilde{\boldsymbol{B}} \boldsymbol{R}^{(i, j)}\left(r_{i, j}\right)
$$

By replacing matrix $\tilde{\boldsymbol{B}}$ by $\tilde{\boldsymbol{B}}^{(\text {new })}$ into criterion (6), the criterion $J_{2}\left(r_{i, j}\right)$ can be expressed as follows:

$$
J_{2}\left(r_{i, j}\right)=\sum_{k=1}^{K} \| \text { off }\left\{\left[\left(\tilde{\boldsymbol{B}}^{(\text {new })}\right)^{\square 2}\right]^{\top} \boldsymbol{C}^{(k,-1)}\left[\left(\tilde{\boldsymbol{B}}^{(\text {new })}\right)^{\square 2}\right]\right\} \|_{F}^{2}
$$

The Hadamard square of $\tilde{\boldsymbol{B}}^{\text {(new) }}$ in (15) can be expressed as a function of $r_{i, j}$ as follows:

$$
\left(\tilde{\boldsymbol{B}}^{\text {(new) }}\right)^{\square 2}=\tilde{\boldsymbol{B}}^{\square 2} \boldsymbol{R}^{(i, j)}\left(r_{i, j}^{2}\right)+2 r_{i, j}\left(\tilde{\boldsymbol{b}}_{i} \boxminus \tilde{\boldsymbol{b}}_{j}\right) \boldsymbol{e}_{j}^{\top}
$$

where $\tilde{\boldsymbol{b}}_{i}$ denotes the $i$-th column of $\tilde{\boldsymbol{B}}$, and $\boldsymbol{e}_{j}$ is the $j$-th column of the identity matrix $I \in \mathbb{R}^{N \times N}$. Inserting (16) into the cost function (15), we have:

$$
\begin{aligned}
& J_{2}\left(r_{i, j}\right)=\sum_{k=1}^{K} \| \text { off }\left(\tilde{\boldsymbol{C}}^{(k, \text { new })}\right) \|_{F}^{2} \\
& =\sum_{k=1}^{K} \| \text { off }\left(\boldsymbol{R}^{(i, j)}\left(r_{i, j}^{2}\right)^{\top} \tilde{\boldsymbol{C}}^{(k)} \boldsymbol{R}^{(i, j)}\left(r_{i, j}^{2}\right)+r_{i, j}^{2} \tilde{c}^{(k, 3)} \boldsymbol{e}_{j} \boldsymbol{e}_{j}^{\top}\right. \\
& \left.+r_{i, j} \boldsymbol{R}^{(i, j)}\left(r_{i, j}^{2}\right)^{\top} \tilde{\boldsymbol{c}}^{(k, 1)} \boldsymbol{e}_{j}^{\top}+r_{i, j} \boldsymbol{e}_{j} \tilde{\boldsymbol{c}}^{(k, 2)} \boldsymbol{R}^{(i, j)}\left(r_{i, j}^{2}\right)\right) \|_{F}^{2}
\end{aligned}
$$

where $\tilde{\boldsymbol{C}}^{(k)}=\tilde{\boldsymbol{A}}^{\top} \boldsymbol{C}^{(k,-1)} \tilde{\boldsymbol{A}}, \tilde{\boldsymbol{c}}^{(k, 1)}=2 \tilde{\boldsymbol{A}}^{\top} \boldsymbol{C}^{(k,-1)}\left(\tilde{\boldsymbol{b}}_{i} \boxminus \tilde{\boldsymbol{b}}_{j}\right)$, $\tilde{\boldsymbol{c}}^{(k, 2)}=\left(\tilde{\boldsymbol{c}}^{(k, 1)}\right)^{\top}$ and $\tilde{c}^{(k, 3)}=4\left(\tilde{\boldsymbol{b}}_{i} \odot \tilde{\boldsymbol{b}}_{j}\right)^{\top} \boldsymbol{C}^{(k,-1)}\left(\tilde{\boldsymbol{b}}_{i} \square \tilde{\boldsymbol{b}}_{j}\right)$ are a matrix, a column vector, a row vector and a scalar of constant values, respectively. (17) shows that just the $j$-th column and row of $\tilde{\boldsymbol{C}}^{(k, \text { new })}$ involve the parameter $r_{i, j}$. Therefore, the minimization of the cost function (17) is equivalent to minimizing the sum of the squares of the $j$-th columns of all the symmetric matrices $\tilde{\boldsymbol{C}}^{(k, \text { new })}$ except their $(j, j)$-th elements. These elements can be expressed by a polynomial function of degree 2 in $r_{i, j}$ as follows, for every $n$ value different of $j$ :

$$
\tilde{C}_{n, j}^{(k, \text { new })}=\tilde{C}_{n, i}^{(k)} r_{i, j}^{2}+\tilde{c}_{n}^{(k, 1)} r_{i, j}+\tilde{C}_{n, j}^{(k)}
$$

where $\tilde{C}_{n, i}^{(k)}$ is the $(n, i)$-th component of $\tilde{\boldsymbol{C}}^{(k)}$, and $\tilde{c}_{n}^{(k, 1)}$ is the $n$-th element of $\tilde{\boldsymbol{c}}^{(k, 1)}$. Then the cost function (17), which is the total sum of squares of (18), is a polynomial function of degree 4 in $r_{i, j}$. The global minimum $r_{i, j}$ is one of the roots of its derivative, which yields the smallest value of (17). Once the optimal $r_{i, j}$ is computed, $\tilde{\boldsymbol{B}}$ is updated by (14) and $\tilde{\boldsymbol{A}}$ is updated by computing $\left(\tilde{\boldsymbol{B}}^{\text {(new) }}\right)^{\square 2}$.

The processing of all the $N(N-1)$ parameters $\theta_{i, j}$ and $r_{i, j}$, is called a $\mathrm{QR}$ sweep. The proposed $\mathrm{JD}_{\mathrm{QR}}^{+}$algorithm is comprised of several $\mathrm{QR}$ sweeps in order to guarantee the convergence. In ICA, when a non-square matrix $\boldsymbol{A} \in \mathbb{R}_{+}^{N \times P}$ with $N>P$ is encountered, we can compress it by a matrix $\boldsymbol{W} \in \mathbb{R}_{+}^{N \times P}$ such that the resulting matrix $\overline{\boldsymbol{A}}=\boldsymbol{W}^{\top} \boldsymbol{A}$ is a nonnegative square matrix [15]. It is noteworthy that the proposed algorithm is different from the two published nonnegative JDC methods, which are based on the LU matrix factorization [10], [11]. We use QR factorization in this paper. The method in [10] estimates $\boldsymbol{B}$ and $\boldsymbol{D}^{(k)}$ alternately, and its performance is sensitive to the initialization. The algorithm in [11] needs to compute the inverse of $\boldsymbol{A}$ in all the $N(N-1)$ Jacobi-like iterations, leading to a high numerical complexity.

\section{Simulation RESULTS}

In this section, the proposed $\mathrm{JD}_{\mathrm{QR}}^{+}$algorithm is compared with several existing JDC methods and BSS algorithms. The performance is measured in terms of the error between the true matrix $\boldsymbol{A}$ and its estimate $\tilde{\boldsymbol{A}}$, as well as the source $s$ and its estimate $\tilde{s}$ when a BSS context is considered. The following scale-invariant and permutation-blind distance is chosen as the preferred measure:

$$
\alpha(\boldsymbol{A}, \tilde{\boldsymbol{A}})=(1 / N) \sum_{n=1}^{N} \min _{\left(n, n^{\prime}\right) \in I_{n}^{2}} d\left(\boldsymbol{a}_{n}, \tilde{\boldsymbol{a}}_{n^{\prime}}\right)
$$

where $\boldsymbol{a}_{n}$ and $\tilde{\boldsymbol{a}}_{n^{\prime}}$ are the $n$-th column of $\boldsymbol{A}$ and the $n^{\prime}$ th column of $\tilde{\boldsymbol{A}}$, respectively. $I_{n}^{2}$ is defined recursively by $I_{1}^{2}=\{1, \cdots, N\} \times\{1, \cdots, N\}$, and $I_{n+1}^{2}=I_{n}^{2}-J_{n}^{2}$, where $J_{n}^{2}=\operatorname{argmin}_{\left(n, n^{\prime}\right) \in I_{n}^{2}} d\left(\boldsymbol{a}_{n}, \tilde{\boldsymbol{a}}_{n^{\prime}}\right)$. In addition, $d\left(\boldsymbol{a}_{n}, \tilde{\boldsymbol{a}}_{n^{\prime}}\right)$ is defined as the pseudo-distance between two vectors [4]:

$$
d\left(\boldsymbol{a}_{n}, \tilde{\boldsymbol{a}}_{n^{\prime}}\right)=1-\left\|\boldsymbol{a}_{n}^{\top} \tilde{\boldsymbol{a}}_{n^{\prime}}\right\|^{2} /\left(\left\|\boldsymbol{a}_{n}\right\|^{2}\left\|\tilde{\boldsymbol{a}}_{n^{\prime}}\right\|^{2}\right)
$$

The smaller the value of (19) is, the better estimation of $\boldsymbol{A}$ is achieved.

\section{A. Simulated semi-nonnegative semi-symmetric arrays}

In this part, $\mathrm{JD}_{\mathrm{QR}}^{+}$is compared with two classic JDC methods, namely ACDC [5] and FFDIAG [6], and one nonnegative JDC method $\mathrm{ACDC}_{\mathrm{LU}}^{+}$[10] with simulated semi-nonnegative semi-symmetric 3 -way arrays $\mathcal{C}$. $\mathcal{C} \in \mathbb{R}^{3 \times 3 \times 5}$ is generated randomly according to equation (2). The loading matrices $\boldsymbol{A}$ and $\boldsymbol{D}$ are randomly drawn from a uniform distribution between 0 and 1 . The pure array $\mathcal{C}$ is perturbed by a semisymmetric residual noise array $\mathcal{V}$. The loading matrices of $\mathcal{V}$ obey the zero-mean unit-variance Gaussian distribution. The resulting noisy 3 -way array can be written by $\mathcal{C}_{N}=$ $\mathcal{C} /\|\mathcal{C}\|_{F}+\sigma_{N} \mathcal{V} /\|\mathcal{V}\|_{F}$, where $\sigma_{N}$ is a scalar controlling the noise level. Then the Signal-to-Noise Ratio (SNR) is defined by $\mathrm{SNR}=-20 \log _{10}\left(\sigma_{N}\right)$. All the algorithms stop either when the relative error of the corresponding criterion between two successive sweeps is less than $10^{-5}$ or when the number of sweeps exceeds 200. We repeat the experiment with SNR ranging from $-10 \mathrm{~dB}$ to $30 \mathrm{~dB}$ with 500 Monte Carlo trials. Figure 1 shows the average curves of $\alpha(\boldsymbol{A}, \tilde{\boldsymbol{A}})$ of all the three algorithms as a function of SNR. It shows that ACDC performs better than FFDIAG under higher SNR levels. The nonnegativity constraint obviously helps $\mathrm{ACDC}_{\mathrm{LU}}^{+}$and $\mathrm{JD}_{\mathrm{QR}}^{+}$ to outperform the classic ones. The proposed $\mathrm{JD}_{\mathrm{QR}}^{+}$algorithm maintains the best estimation accuracy, especially for the lower SNR levels.

\section{B. BSS application on MRS data}

In this section, the BSS performance of $\mathrm{JD}_{\mathrm{QR}}^{+}$is compared with an effective ICA method $\mathrm{CoM}_{2}$ [16] and a Nonnegative Matrix Factorization (NMF) method based on alternating NonNegativity Least Squares (NNLS) [17], through an experiment carried out on simulated MRS data. Two metabolites, namely the Choline and Myo-inositol, serve as source signals $s_{1}(f)$ and $s_{2}(f) .32$ observations are generated according to the noisy linear mixing model $\boldsymbol{x}(f)=\boldsymbol{A} \boldsymbol{s}(f)+\boldsymbol{\nu}(f)$, where $\boldsymbol{\nu}(f)$ is an additive white Gaussian noise. $\boldsymbol{A} \in \mathbb{R}_{+}^{32 \times 2}$ is similarly generated as in the previous section. For an ICA method based 


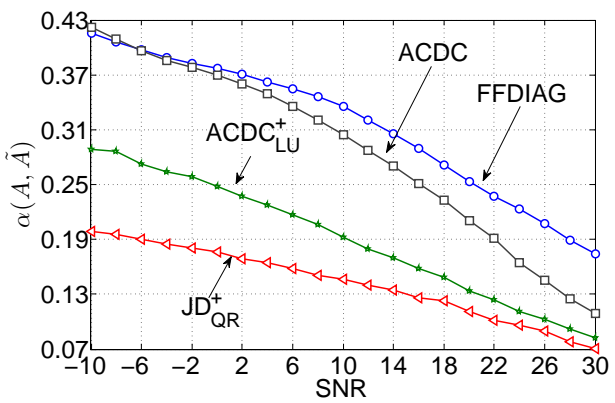

Fig. 1. Average error $\alpha(\boldsymbol{A}, \tilde{\boldsymbol{A}})$ evolution of ACDC, FFDIAG, $\mathrm{ACDC}_{\mathrm{LU}}^{+}$and $\mathrm{JD}_{\mathrm{QR}}^{+}$as a function of SNR on simulated arrays.

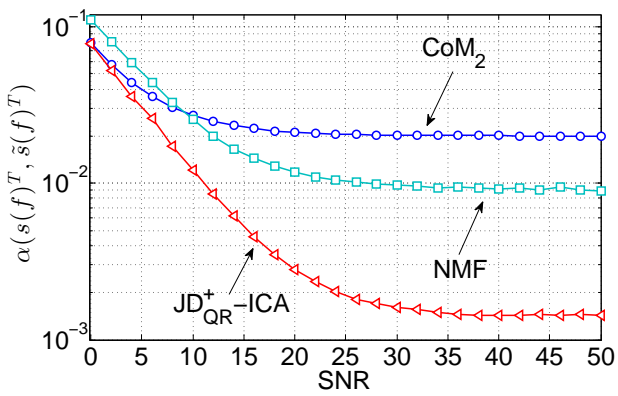

Fig. 2. Average error $\alpha\left(\{\boldsymbol{s}(f)\}^{\top},\{\tilde{\boldsymbol{s}}(f)\}^{\top}\right)$ evolution of $\mathrm{CoM}_{2}$, NMF and $\mathrm{JD}_{\mathrm{QR}}^{+}$-ICA as a function of SNR for BSS of 2 simulated MRS metabolites.

on $\mathrm{JD}_{\mathrm{QR}}^{+}$, namely $\mathrm{JD}_{\mathrm{QR}}^{+}-\mathrm{ICA},\{\boldsymbol{x}(f)\}$ is compressed by means of a matrix $\boldsymbol{W} \in \mathbb{R}_{+}^{32 \times 2}$ computed using the method proposed in [15], such that the number of observations is reduced to 2. The 3-way array $\mathcal{C}$ is built by stacking four 4 -th order cumulant matrix slices. We repeat the experiment with SNR ranging from $0 \mathrm{~dB}$ to $50 \mathrm{~dB}$ with 200 Monte Carlo trials. The average curves of the estimating error $\alpha\left(\{\boldsymbol{s}(f)\}^{\top},\{\tilde{\boldsymbol{s}}(f)\}^{\top}\right)$ of all the three methods as a function of SNR are shown in figure 2. It shows that the proposed $\mathrm{JD}_{\mathrm{QR}}^{+}-\mathrm{ICA}$ algorithm maintains competitive advantages when SNR $\geq 5 \mathrm{~dB}$. Figure 3 shows the separation results of all the methods with a SNR of 10 $\mathrm{dB}$ for one typical realization. Regarding $\mathrm{CoM}_{2}$ and NMF, there are some obvious disturbances presented in the estimated metabolites. As far as $\mathrm{JD}_{\mathrm{QR}}^{+}-\mathrm{ICA}$ is concerned, the estimated source metabolites are quasi-perfect.

\section{CONCLUSION}

In this paper, we have addressed the problem of the $\mathrm{CP}$ decomposition of semi-nonnegative semi-symmetric 3-way arrays. We proposed a method, called $\mathrm{JD}_{\mathrm{QR}}^{+}$, based on the $\mathrm{QR}$ factorization of the Hadamard square root of the nonnegative loading matrix. A numerical experiment on simulated arrays highlights its advantage. A BSS application on MRS signals also demonstrates the interest of the proposed method.

\section{REFERENCES}

[1] X. Luciani and L. Albera, "Canonical polyadic decomposition based on joint eigenvalue decomposition," Chemometr. Intell. Lab., vol. 132, pp. 152-167, 2014.

[2] A. Smilde, R. Bro, and P. Geladi, Multi-way Analysis: Applications in the Chemical Sciences. West Sussex, England: Wiley, 2004.

[3] P. Comon, X. Luciani, and A. L. F. de Almeida, "Tensor decompositions, alternating least squares and other tales," J. Chemometr., vol. 23, pp. 393-405, 2009.

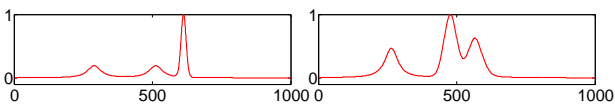

(a) Choline and Myo-inositol source metabolites

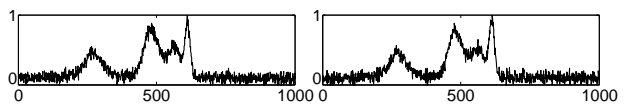

(b) Two of the observations

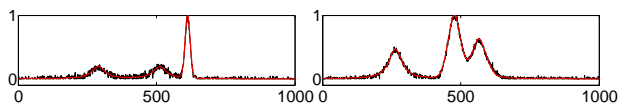

(c) Estimates by $\mathrm{JD}_{\mathrm{QR}}^{+}-\mathrm{ICA}$

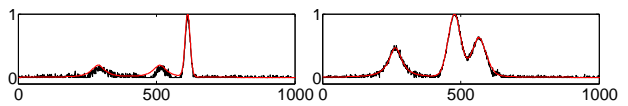

(d) Estimates by $\mathrm{CoM}_{2}$

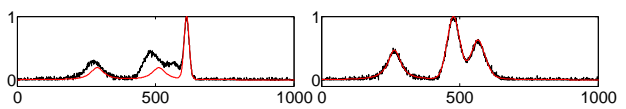

(e) Estimates by NMF

Fig. 3. MRS source metabolites, observations and estimated metabolites by $\mathrm{JD}_{\mathrm{QR}}^{+}-\mathrm{ICA}, \mathrm{CoM}_{2}$ and NMF with $\mathrm{SNR}=10 \mathrm{~dB}$ for one typical realization. The overlapping red lines in figures (c), (d) and (e) indicate the correct sources.

[4] L. Albera, A. Ferréol, P. Comon, and P. Chevalier, "Blind identification of overcomplete mixtures of sources (BIOME)," Linear Algebra Appl., vol. 391, pp. 3-30, 2004.

[5] A. Yeredor, "Non-orthogonal joint diagonalization in the least-squares sense with application in blind source separation," IEEE Trans. Signal Process., vol. 50, no. 7, pp. 1545-1553, 2002.

[6] A. Ziehe, P. Laskov, G. Nolte, and K.-R. Muller, "A fast algorithm for joint diagonalization with non-orthogonal transformations and its application to blind source separation," J. Mach. Learning Res., vol. 5, pp. 777-800, 2004.

[7] A. Cichocki, R. Zdunek, A. H. Phan, and S. Amari, Nonnegative Matrix and Tensor Factorizations: Applications to Exploratory Multi-way Data Analysis and Blind Source Separation. West Sussex, United Kingdom: WILEY, 2009.

[8] A. Shashua, R. Zass, and T. Hazan, "Multi-way clustering using super-symmetric non-negative tensor factorization," in Computer VisionECCV, 2006, pp. 595-608.

[9] P. Tichavský and Z. Koldovský, "Weight adjusted tensor method for blind separation of underdetermined mixtures of nonstationary sources," IEEE Trans. Signal Process., vol. 59, no. 3, pp. 1037-1047, 2011.

[10] L. Wang, L. Albera, A. Kachenoura, H. Shu, and L. Senhadji, "Nonnegative joint diagonalization by congruence based on LU matrix factorization," IEEE Signal Process. Lett., vol. 20, no. 8, pp. 807-810, 2013.

[11] L. Wang, L. Albera, H. Shu, and L. Senhadji, "A new Jacobi-like nonnegative joint diagonalization by congruence," in Proceedings of the XXI European Signal Processing Conference (EUSIPCO'13), 2013, paper id: 1569744591.

[12] A. Souloumiac, "Nonorthogonal joint diagonalization by combining Givens and hyperbolic rotations," IEEE Trans. Signal Process., vol. 57, no. 6, pp. 2222-2231, 2009.

[13] M. Chu, F. Diele, R. Plemmons, and S. Ragni, "Optimality computation and interpretation of non negative matrix factorizations," Wake Forest University, Tech. Rep., 2004.

[14] J.-P. Royer, P. Comon, and N. Thirion-Moreau, "Nonnegative 3-way tensor factorization via conjugate gradient with globally optimal stepsize," in ICASSP'11, 2011, pp. 4040-4043.

[15] L. Wang, A. Kachenoura, L. Albera, H. Shu, and L. Senhadji, "Nonnegative compression for semi-nonnegative independent component analysis," submitted to IEEE SAM 2014 Workshop.

[16] P. Comon, "Independent component analysis, a new concept?" Signal Process., vol. 36, no. 3, pp. 287-314, 1994.

[17] H. Kim and H. Park, "Nonnegative matrix factorization based on alternating nonnegativity constrained least squares and active set method," SIAM J. Matrix Anal. Appl., vol. 30, no. 2, pp. 713-730, 2008. 